\title{
Dynamics of HSP60-Cypd Binding Studied with Surface Plasmon Resonance
}

Ekaterina A Korobkova*

Department of Sciences, John Jay College of Criminal Justice at the City University of New York, New York, NY 10019

\begin{abstract}
Objectives: The binding of chaperonin HSP60 to cyclophilin D (CypD) represents an oncogenic pathway that prevents mitochondria from undergoing permeability transition pore (PTP) opening. Thus HSP60 may be considered as an attractive target for the design of chemical inhibitors. The complexity of the HSP60 structure prevents the use of standard screening methods. The present study was aimed to analyze the dynamics of CypD interactions with different HSP60 domains.
\end{abstract}

Method: Surface plasmon resonance (SPR) technology was employed. Antibodies that map to various regions of the HSP60 were immobilized on a CM5 biosensor chip using amino-coupling chemistry. HSP60 was attached to various antibodies on the chip resulting in different orientations of the protein, and the kinetics of its binding to HSP60 was analyzed.

Results: The dissociation rate constants for HSP60-CypD interactions ranged between $5.5 \times 10^{-4} \mathrm{~s}^{-1}$ and $16 \times$ $10^{-4} \mathrm{~s}^{-1}$. The dissociation equilibrium constants varied from $15.8 \mathrm{nM}$ to $43.5 \mathrm{nM}$. An antibody recognizing a region between residues 50 and 100 in the equatorial domain of HSP60 prevented its association with CypD.

Conclusion: SPR technology proved successful in the analysis of the interactions between CypD and HSP60 subunits. The binding strength was comparable to that of a relatively strong antibody-antigen binding. The preferential binding of CypD to a specific domain within HSP60 subunit suggests the possibility of designing a molecular antagonist.

Keywords: Surface plasmon resonance; HSP60; CypD; Apoptosis; Mitochondria; PTP opening; Kinetics

Abbreviations: CypD: Cyclophilin D; HSP60: Heat Shock Protein 60; Ppis: Peptidyl-Prolyl Cis-Trans Isomerase; PTP: Permeability Transition Pore; SPR: Surface Plasmon Resonance

\section{Introduction}

In the last ten years, extensive proteomic analysis has been performed on the mitochondria of various types of cancer cells [1]. One of the proteins found selectively overexpressed in the mitochondria of cancer cells is chaperone HSP60. The elevated levels of HSP60 were detected in the mitochondria of myeloid leukemia [2], breast [3], prostate [4-6], colorectal [6,7], gastric [8], and lung cancer [6].

At normal conditions, HSP60 is located in the cytoplasm and mitochondria. When mammalian cells are exposed to fatal conditions, HSP60 is quickly transferred into mitochondria by the mechanisms involving HSP70 [9]. HSP60 exhibits protective effects against mitochondrial abnormalities [10], neurodegenerative pathologies [11], and ischemic injury [12]. A high degree of similarity in the amino acid sequence between HSP60 and E. coli protein GroEL indicates that HSP60 is a mammalian homolog of GroEL [13]. Electron microscopic analysis showed that both HSP60 and GroEL protein contain 14 subunits assembled in two 7 -fold rings $[13,14]$. The whole complex has a shape of a porous cylinder. The crystal structure of the bacterial chaperonin GroEL shows that each subunit in the 14-mer complex consists of three domains [15]: a large equatorial domain that forms the central part of the cylinder, an apical domain that forms the edges of the cylinder, and an intermediate domain that links the equatorial and apical domains. HSP60 and GroEL also exhibit physiological properties in common. The level of these proteins increases drastically at elevated temperatures. Both HSP60 and GroEL possess ATPase activity and assist other proteins in folding and the formation of oligomers [16].
Mitochondrial HSP60 is known to play an essential role in apoptosis through the association with other proteins. HSP60 binds hepatitis B virus $\mathrm{X}$ protein and assists with apoptosis induced by this protein [17]. The chaperone binds pro-caspase- 3 and facilitates its maturation thus controlling one of the steps in the apoptotic program [18]. In contrary, there is evidence of the antiapoptotic and cancer-stimulating activity of HSP60. It forms a complex with a tumor suppressor protein p53 inhibiting its activity. HSP60 also associates with survivin, apoptosis inhibitor, stabilizing it and facilitating cellular growth [6]. HSP60 supports the accumulation of hypoxia-inducible factor (HIF-1a), a protein identified as a target in cancer therapy [19]. Recently, Ghosh et al. found that HSP60 forms a complex with immunophilin CypD [20]. Notably, this association occurs selectively in the mitochondria of cancer cells as opposed to normal cells. The authors validated the HSP60-CypD interaction as a potential target in cancer therapy by demonstrating that the genetic targeting of HSP60 led to CypDdependent mitochondrial PTP opening, apoptosis, and the inhibition of the growth of glioblastoma [20].

CypD is located on the inner membrane and protrudes to the matrix of mitochondria. The exact mechanism of the CypD-mediated

*Corresponding author: Ekaterina A Korobkova, Department of Sciences, John Jay College of Criminal Justice at the City University of New York, 524 W 59th St., New York, NY 10019, USA, Tel: (212) 237-8064; Fax: 212-237-8318; E-mail: ekorobkova@jjay.cuny.edu

Received October 02, 2015; Accepted October 30, 2015; Published October 31 , 2015

Citation: Korobkova EA (2015) Dynamics of HSP60-Cypd Binding Studied with Surface Plasmon Resonance. Pharm Anal Acta 6: 432. doi:10.4172/21532435.1000432

Copyright: $\odot 2015$ Korobkova EA. This is an open-access article distributed under the terms of the Creative Commons Attribution License, which permits unrestricted use, distribution, and reproduction in any medium, provided the original author and source are credited. 
PTP control is not understood, however it is suggested that its peptidylprolyl cis-trans isomerase (PPIs) activity facilitates a conformational change of pore-forming components located in the inner membrane [21].

To study the kinetics of HSP60-CypD binding, we performed SPR using CM5 chip and aminocoupling technique. We determined the kinetic parameters of HSP60-CypD interactions at different orientations of HSP60 monomer on the chip surface employing five antibodies recognizing various domains on HSP60. An antibody specifically recognizing an equatorial domain of HSP60 significantly weakened the association between the proteins. We hypothesize that the binding of HSP 60 to CypD in the area of the equatorial domain may lead to the conformational changes in $\mathrm{CypD}$, which in turn prevents the PTP opening and inhibits apoptosis.

\section{Materials and Method}

Recombinant HSP60 protein was purchased from Sino Biological Inc (North Wales, PA). HSP60 antibodies were purchased from Novus Biologicals Inc (Littleton, CO). CypD was purchased from ProSpec Inc (East Brunswick, NJ). The following antibodies were purchased from Novus Biologicals: NBP1-77396 (residues 300-360, antibody 1), NBP177397 (residues 70-150, antibody 2), NBP1-47309 (residues 50-100, antibody 3), NBP1-47310 (residues 250-300, antibody 4), and NB10091819 (residues surrounding Threonine 547, antibody 5).

Surface Plasmon Resonance (SPR) was performed using the Biacore X system (GE Healthcare). In SPR system, molecules under investigation (ligands) are attached to the surface of a sensor chip, while other binding molecules (analytes) are passed over the surface in a mobile phase. The interactions of the molecules are then monitored in real time. The signal is based on the changes in the refractive index of the surface due to the variations of the mass of the molecules occurring as a result of binding and dissociation of the analyte.

An antibody was immobilized onto a carboxymethyl dextran biosensor chip, CM5, using amine coupling. The surface was equilibrated by applying a continuous flow of water at the rate of $10 \mu \mathrm{l} /$ min. The dextran matrix was activated with an 8 min injection of a water solution containing $0.2 \mathrm{M}$ of 1-ethyl-3-(3-dimethylaminopropyl)carbodiimide (EDC) and $0.05 \mathrm{M}$ of $\mathrm{N}$-hydroxysuccinimide (NHS). After the activation, $0.1 \mathrm{mg} / \mathrm{ml}$ solution of antibody was applied to the surface in a $10 \mathrm{mM}$ sodium acetate buffer ( $\mathrm{pH}$ 5.0) for $8 \mathrm{~min}$. To inactivate residual esters, the surface was exposed to $1 \mathrm{M}$ ethylamine$\mathrm{HCl}$ ( $\mathrm{pH} 8.5$ ), for $9 \mathrm{~min}$. After the deactivation, the chip surface was re-equilibrated in Hepes buffer ( $\mathrm{pH}$ 7.4) containing $10 \mathrm{mM}$ Hepes, $150 \mathrm{mM} \mathrm{NaCl}$, and $2 \mathrm{mM}$ EDTA. The chip was kept under the flow of the Hepes buffer for at least 24 hours. A long running time was necessary for the effective equilibration of the antibody moiety on the chip surface and acquiring a proper antibody's conformation. HSP60 protein at a concentration of $580 \mathrm{nM}$ in $10 \mathrm{mM}$ Hepes buffer ( $\mathrm{pH} 7.4)$ was injected at a flow rate of $10 \mu \mathrm{l} / \mathrm{min}$ over the immobilized antibody. A $280 \mathrm{nM}$ solution of CypD was injected at a flow rate of $10 \mu \mathrm{l} / \mathrm{min}$ over the immobilized HSP60, when the RU level was stabilized (45 min after the injection of HSP60 was completed). The chip surface with antibody covalently attached to it was regenerated by applying a solution of $10 \mathrm{mM}$ solution of sodium hydroxide for $7 \mathrm{~min}$. The schematic presentation of the experimental design is show in Figure 1.

The sensograms were analyzed using the Biacore software. The base level was subtracted from the curves such that the signal at the beginning of the injection of HSP60 was zero. The fits were performed by using the "Kinetics Separate $k_{a} / k_{d}$ " feature. The dissociation and association curves from each sensogram were analyzed separately using the following equations:

$$
\begin{array}{ll}
R=R_{0} \times e^{-k_{d} \times\left(\mathrm{t}-\mathrm{t}_{0}\right)}+\text { offset } & \text { Equation 1 } \\
R=\frac{k_{a} \times[\mathrm{cypD}] \times \mathrm{R}_{\max }}{k_{a} \times[\mathrm{cypD}]+k_{d}} \times\left(1-\mathrm{e}^{-\left(\mathrm{k}_{a} \times[\mathrm{cypD}]+\mathrm{k}_{d}\right) \times\left(\mathrm{t}-\mathrm{t}_{0}\right)}\right)+\mathrm{RI} & \text { Equation 2 }
\end{array}
$$

The dissociation curve was analyzed first. The fit was started at the point corresponding to $50 \mathrm{~s}$ after the protein injection. The dissociation fitting interval was $100 \mathrm{~s}$. The association curve was fitted over the whole range of protein binding. The $k_{d}$ value obtained from the first fit was used as a parameter in the analysis of the association curve. The concentration values of $5.8 \times 10^{-7}$ and $2.8 \times 10^{-7}$ were used for evaluating the kinetics of HSP60 binding to antibody and CypD binding to HSP60, respectively. As a result of the analysis of the association curve, the values of $k_{a}$ and $K_{D}=k_{d} / k_{a}$ were obtained. The standard errors for $k_{a}$ and $k_{d}$ were obtained with the values of the constants. The standard error for $K_{D}$ was calculated by using a formula for the standard deviation of a ratio of two variables. The sensograms were measured three times for each antibody. The average values of $k_{d}$ and $K_{D}$ were calculated from three experiments, and their errors were determined accordingly.

\section{Results and Discussion}

The interactions between HSP60 and CypD were studied with SPR technique. The antibodies were chosen such that they specifically bound to all major parts of the $64 \mathrm{kDa}$ subunit of HSP60. Experimental evidence suggests that HSP60 protein is structurally homologous to the bacterial GroEL protein $[13,14]$. Based on this structural similarity, the mapping regions of the HSP60 correspond to the apical domain (antibody 1), equatorial-intermediate domains (antibody 2), equatorial domain (antibody 3), and apical domain (antibody 4). The location of the C-terminal, a region specifically bound to antibody 5 (around residue 547), could not be very well deduced from the analysis of the crystal structure. Braig et al. suggested that the C-terminal tail protrudes towards the interior of the protein cylinder [15].

The antibodies were attached to the CM5 chips using an aminocoupling procedure as described in "Materials and methods". The sensogram demonstrating the signal variation associated with the addition of NHS/EDC, antibody 1 , and ethanolamine onto the chip is shown in Figure 2A. It can be seen that the attachment of the antibody to the chip surface is very efficient, even though the treatment with basic solution removes some portion of covalently attached antibody from the chip. The sensograms associated with HSP60 binding to and dissociation from the antibodies were analyzed (Figure $2 \mathrm{~B}$ ), the values of the equilibrium dissociation constants $\left(K_{D}\right)$ of HSP60antibody binding varied between 11 and $23 \mathrm{nM}$ (Table 1) indicating very strong antigen-antibody interactions. It is noteworthy that the binding strength between HSP60 and the respective antibodies

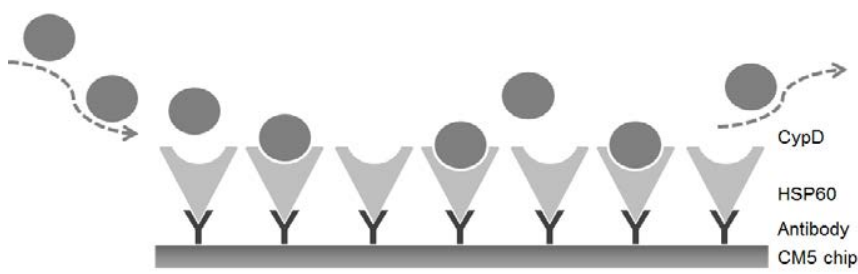

Figure 1: Schematic presentation of CM5 chip with attached HSP60antibody, HSP60 (ligand), and CypD (analyte) passing over the chip surface. 

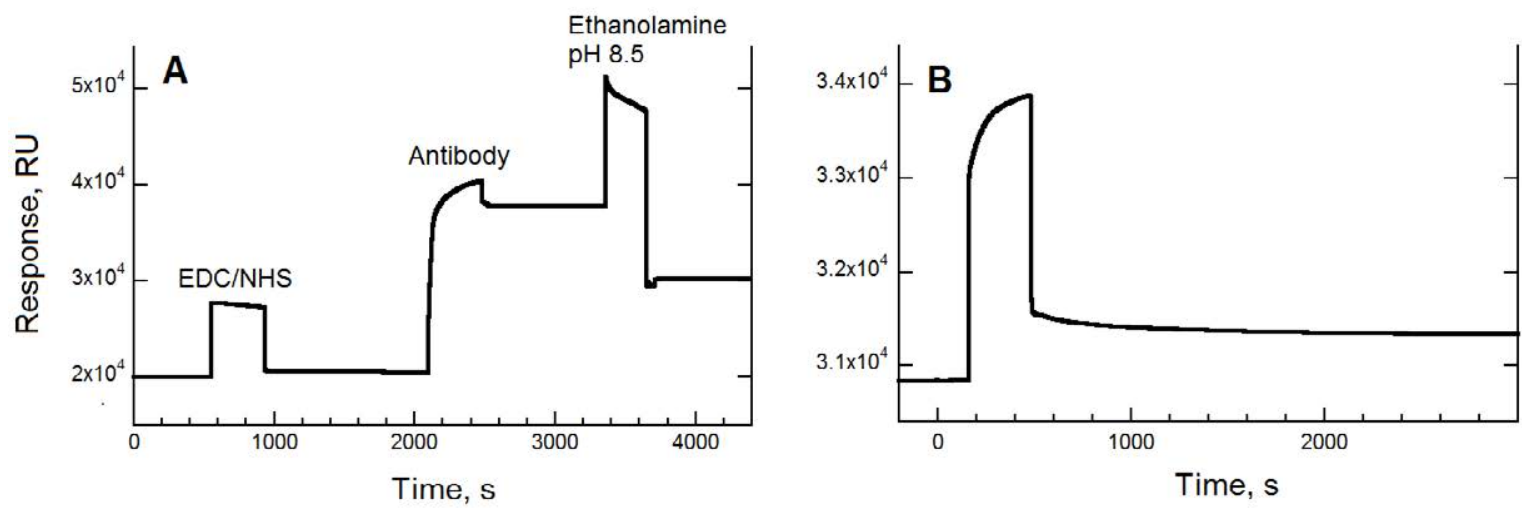

Figure 2: Biacore sensograms illustrating the attachment of HSP60 to CM5 chip via HSP60-specific antibody. (A) The immobilization of the antibody 1 to the CM5 chip surface. The chip was equilibrated in deionized water at a rate of $10 \mu \mathrm{l} / \mathrm{min}$. The sensogram demonstrates (1) the injection of a mixture of EDC/NHS, (2) the injection of the antibody, and (3) the deactivation of the unreacted sucinimide esters with a basic solution of ethanolamine. The antibody was prepared in a $10 \mathrm{mM}$ sodium acetate buffer $(\mathrm{pH} \mathrm{5})$ at a concentration of $0.1 \mathrm{mg} / \mathrm{ml}$. After the injection of ethanolamine, the chip was re-equilibrated in $10 \mathrm{mM}$ Hepes buffer ( $\mathrm{pH} 7.4$ ) for 24 hours. (B) The binding of HSP60 to the antibody immobilized on the CM5. A $0.580 \mu \mathrm{M}$ solution of HSP60 in $10 \mathrm{mM}$ hepes buffer (pH 7.4 ) was injected at a rate of 10 $\mu \mathrm{l} / \mathrm{min}$ for $8 \mathrm{~min}$. $500 \mathrm{RU}$ of the protein was attached.

\begin{tabular}{|c|c|c|c|}
\hline Antibody \# & HSP60 residues & HSP60 domain & apical \\
\hline 1 & $300-360$ & M & $1.99 \times 10^{-8} \pm 1.62 \times 10^{-10}$ \\
\hline 2 & $70-150$ & equatorial-intermediate & $2.32 \times 10^{-8} \pm 1.94 \times 10^{-10}$ \\
\hline 3 & $50-100$ & equatorial & $1.12 \times 10^{-8} \pm 2.94 \times 10^{-10}$ \\
\hline 4 & $250-300$ & apical & $1.80 \times 10^{-8} \pm 6.20 \times 10^{-10}$ \\
\hline 5 & around 547 & $1.45 \times 10^{-8} \pm 1.03 \times 10^{-10}$ & - \\
\hline
\end{tabular}

Table 1: The average values of the dissociation constants for the interactions between HSP60 and five different antibodies selectively recognizing different parts of the protein's domains.

was approximately the same for all five antibodies, which makes the comparison between various orientations of the protein reliable.

The sensograms representing the variations of RU signal as a result of CypD-HSP60 binding are shown in Figure 3. It can be seen that HSP60 attached to the antibodies 1, 2, 4, and 5 retains CypD very efficiently. This is indicated by very small slopes of the dissociation curves and the steady levels of the CypD signal observed at long times after the protein injection. However, in the case of the antibody 3 CypD completely dissociates from the chip surface with immobilized HSP60 (Figure 3). The sensogram for each antibody was run at least three times. The analysis of the dynamics of the interactions between the two proteins led to consistent data within the same antibodies (Tables 2 and 3).

An example of the analyzed curve is shown in Figure 4. The values of $k_{d}$ and $K_{D}$ obtained from the individual experiments as well as their averages are presented in Tables 2 and 3, respectively. The values of $k_{d}$ ranged between $5.50 \times 10^{-4} \mathrm{~s}^{-1}$ and $16.0 \times 10^{-4} \mathrm{~s}^{-1}$ (Table 2), and the values of $K_{D}$ ranges between $15.8 \mathrm{nM}$ and $43.5 \mathrm{nM}$ (Table 3).

According to the crystal structure of GroEL, a bacterial analog of HSP60, the protein assembles into a porous cylinder consisting of two rings. Each ring is made of 7 identical monomers, and the rings are stacked together in a dyad symmetry. Each monomer consists of three major parts, an apical domain constituting the edges of the cylinder, an equatorial domain that forms the waist of the cylinder, and an intermediate domain that links the other two domains together (Figure 5) [15].

Fenton et al. performed mutation analysis to determine sites on GroEL protein responsible for its chaperonin function [22]. The data indicated that unfolded polypeptides attach to the central channel of the cylinder, specifically the binding occurs via hydrophobic amino acids on the protein's inside face of the apical domain (residues 199, 203, 204, 234, 237, 259, 263, and 264). Protein segments facing the central channel and segments located on the top surface of the cylinder are poorly resolved in the crystal structures and have high temperature factors. The authors suggested that this region could be intrinsically adaptable to a wide range of unfolded polypeptides rather than to fully folded proteins. Our data indicate that the binding of CypD to HSP60 is very strong when HSP60 is attached to the chip surface via its apical domain (antibody 1 and antibody 4), while the binding of CypD is much weaker when HSP60 is attached to the chip via its equatorial domain. As indicated in Tables 2 and 3, the values of $k_{d}$ and $K_{D}$ are at least two-fold greater in the case of the equatorial orientation of HSP60 than those determined with the protein's apical orientation. This result suggests a preferential binding of a fully folded protein CypD to the regions of the equatorial domain on HSP60 rather than to its apical domain

Cheng et al. investigated the kinetics of the formation of the HSP60 14-mer assembly and concluded that there must be a pre-existing fully folded HSP60 oligomer in order to catalyze the assembly of new 14-mer complex [23]. The authors excluded the possibility of selfassembly of HSP60 monomers. In the present work, HSP60 monomers were attached to the chip surface. In this case, various parts of the protein are almost equally accessible by the antibodies specifically recognizing different segments of the protein. This is supported by the values of HSP60-antibody binding constants (Table 1). The SPR data indicate that out of the five HSP60 antibodies explored antibody 3 , which recognizes a region in between residues 50 and 100 (a part of the equatorial domain), causes a complete dissociation of CypD from HSP60 and thus antagonizes the binding. Equatorial domain 
Citation: Korobkova EA (2015) Dynamics of HSP60-Cypd Binding Studied with Surface Plasmon Resonance. Pharm Anal Acta 6: 432. doi:10.4172/21532435.1000432
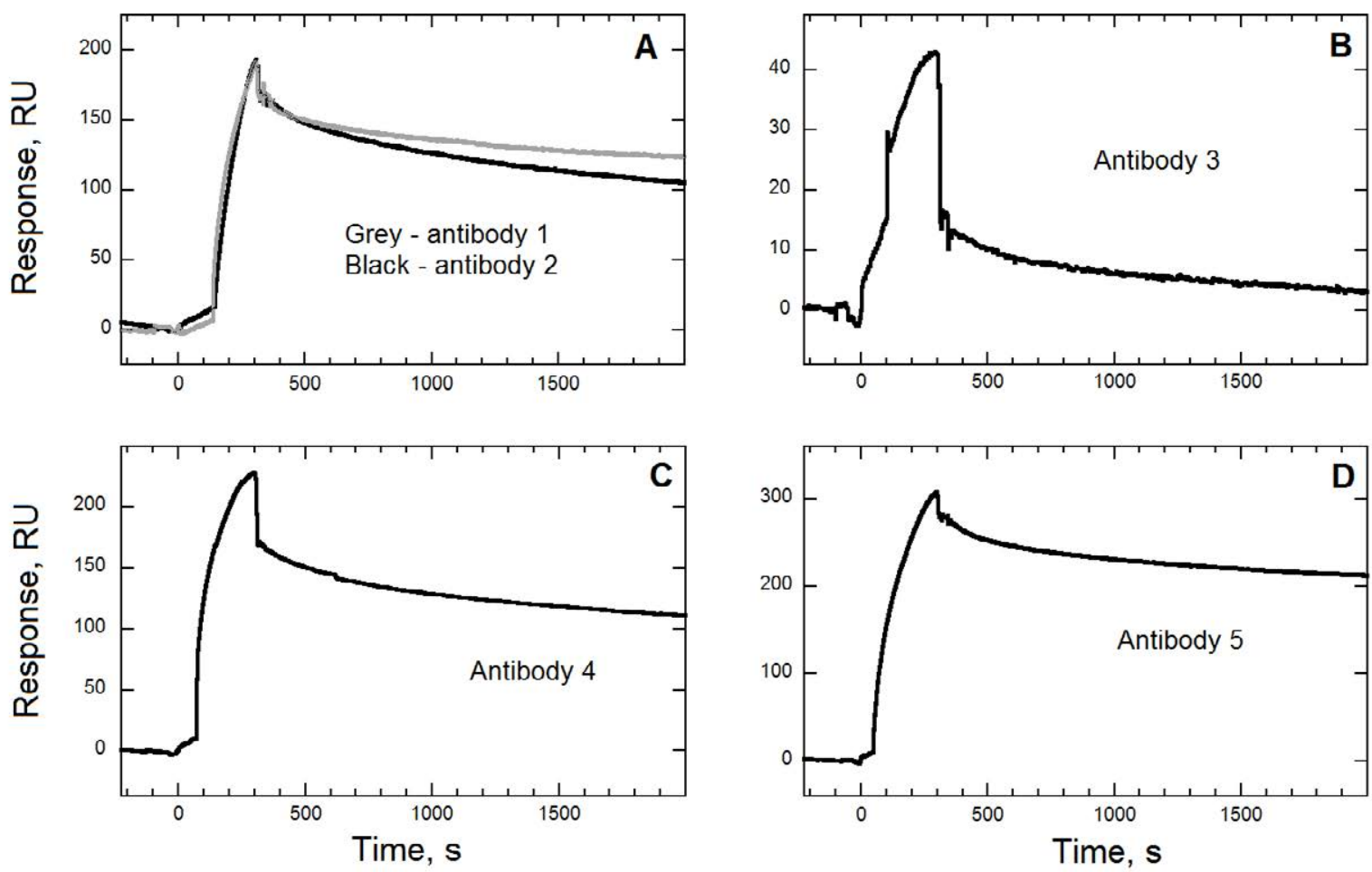

Figure 3: The binding of CypD to HSP60. A solution of CypD in $10 \mathrm{mM}$ Hepes buffer (pH 7.4) was passed over sensor chips on which HSP60 was immobilized Retention of the protein on the sensor chip was indicated by a change in RU over the course of the 300 s injection interval. (A) Grey line - a sensogram illustrating the interactions between CypD and HSP60 attached to antibody 1 (residues 300-360), black line - binding of CypD to HSP60 attached to antibody 2 (residues 70-150); (B) binding of CypD to HSP60 attached to antibody 3 (residues 50-100); (C) binding of CypD to HSP60 attached to antibody 4 (residues 250-300); (D) binding of CypD to HSP60 attached to antibody 5 (around a residue 547). The concentration of the injected solution of CypD was $280 \mathrm{nM}$, and the injection time was 5 min.

\begin{tabular}{|c|c|c|c|c|}
\hline & Antibody 1 & Antibody 2 & Antibody 3 & Antibody 5 \\
\hline Exp. 1 & $6.39 \times 10^{-4} \pm 3.06 \times 10^{-5}$ & $6.96 \times 10^{-4} \pm 1.57 \times 10^{-5}$ & $1.96 \times 10^{-3} \pm 1.11 \times 10^{-4}$ & $9.98 \times 10^{-4} \pm 6.78 \times 10^{-5}$ \\
\hline Exp. 2 & $4.57 \times 10^{-4} \pm 6.97 \times 10^{-6}$ & $7.27 \times 10^{-4} \pm 9.89 \times 10^{-6}$ & $1.83 \times 10^{-3} \pm 7.99 \times 10^{-5}$ & $5.51 \times 10^{-4} \pm 2.20 \times 10^{-5}$ \\
\hline Exp. 3 & $5.54 \times 10^{-4} \pm 2.38 \times 10^{-5}$ & $9.84 \times 10^{-4} \pm 2.00 \times 10^{-5}$ & $1.00 \times 10^{-3} \pm 4.34 \times 10^{-5}$ & $6.31 \times 10^{-4} \pm 8.94 \times 10^{-6}$ \\
\hline Avg & $5.50 \times 10^{-4} \pm 1.31 \times 10^{-5}$ & $8.02 \times 10^{-4} \pm 9.09 \times 10^{-6}$ & $16.0 \times 10^{-4} \pm 4.78 \times 10^{-5}$ & $7.34 \times 10^{-4} \pm 2.40 \times 10^{-5}$ \\
\hline
\end{tabular}

Table 2: The values of the dissociation constants, $k_{d}, \mathrm{~s}^{-1}$, of HSP60-CypD interactions measured with different orientations of HSP60 immobilized on the antibodies.

\begin{tabular}{|c|c|c|c|c|}
\hline & Antibody 1 & Antibody 2 & Antibody 3 & Antibody 5 \\
\hline Exp. 1 & $1.92 \times 10^{-8} \pm 9.48 \times 10^{-10}$ & $2.30 \times 10^{-8} \pm 6.07 \times 10^{-10}$ & $4.14 \times 10^{-8} \pm 3.07 \times 10^{-9}$ & $1.63 \times 10^{-8} \pm 1.15 \times 10^{-9}$ \\
\hline Exp. 2 & $1.33 \times 10^{-8} \pm 3.16 \times 10^{-10}$ & $3.09 \times 10^{-8} \pm 5.64 \times 10^{-10}$ & $6.63 \times 10^{-8} \pm 3.70 \times 10^{-9}$ & $2.04 \times 10^{-8} \pm 5.78 \times 10^{-10}$ \\
\hline Exp. 3 & $2.11 \times 10^{-8} \pm 9.45 \times 10^{-10}$ & $3.83 \times 10^{-8} \pm 1.04 \times 10^{-9}$ & $2.27 \times 10^{-8} \pm 1.09 \times 10^{-9}$ & $1.88 \times 10^{-8} \pm 3.36 \times 10^{-10}$ \\
\hline Avg & $1.79 \times 10^{-8} \pm 4.58 \times 10^{-10}$ & $3.07 \times 10^{-8} \pm 4.44 \times 10^{-10}$ & $4.35 \times 10^{-8} \pm 2.46 \times 10^{-9}$ & $1.27 \times 10^{-8} \pm 1.47 \times 10^{-10}$ \\
\hline
\end{tabular}

Table 3: The values of the dissociation rate constants, $K_{D}, M$, of HSP60-CypD interactions measured with different orientations of HSP60 immobilized on the antibodies.

forms a foundation of the HSP60 14-mer assembly having cylindrical shape (Figure 5). The two rings in the cylinder are connected via their equatorial domains [15]. The equatorial domain of one HSP60 subunit does not contact the apical and intermediate domains of the adjacent subunits, and the essential part of the domain is accessible by solvent components. Interestingly, the $K_{D}$ value measured with antibody 2 recognizing parts of both intermediate and equatorial domains is only 1.4 times lower than that measured with antibody 3 , while other $K_{D}$ values are at least 2.3 times lower (Table 3 ). The intermediate domain forms covalent connections between the equatorial and apical domains within the same subunit. Moreover, the intermediate domain is attached non-covalently to the apical domain of the adjacent subunit (Figure 5). Thus the intermediate domain facilitates the allosteric communication between the domains within the same subunit and between different subunits of HSP60. We hypothesize that the binding of CypD protein in the area of equatorial domain adjacent to the intermediate domain of the HSP60 subunit may stimulate allosteric modifications that could spread though the neighboring subunits affecting the conformation and the PPIs activity of CypD attached to the other HSP60 subunits. These cooperative allosteric adjustments within the HSP60-CypD compex could modulate PTP opening.

The majority of protein-protein interfaces involved in oncogenic signaling pathways is not druggable due to a flat shape or a large area [24]. A target may be considered druggable with small molecules if its surface accessible to the solvent possesses a pocket lined with hydrophobic amino acid side chains [25]. In an earlier study, Nagamuto et al. found that epolactaene tertiary butyl ester (ETB) 
Citation: Korobkova EA (2015) Dynamics of HSP60-Cypd Binding Studied with Surface Plasmon Resonance. Pharm Anal Acta 6: 432. doi:10.4172/21532435.1000432

Page 5 of 6

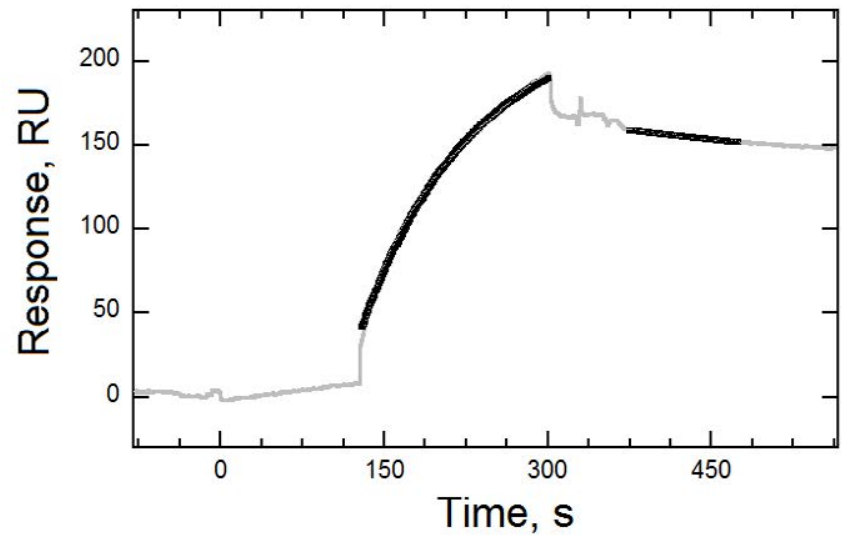

Figure 4: Biacore sensogram depicting the interactions between HSP60 and CypD. HSP60 is immobilized on the chip surface via antibody 1. The grey line shows the RU signal as the solution of CypD passes over the chip. The black lines represent the fitting curves of the association and dissociation parts of the sensogram. The $k_{d}$ value is $4.57 \times 10^{-4} \mathrm{~s}^{-1}$, and the $k_{\mathrm{a}}$ value is $3.44 \times 10^{4}$ $\mathrm{M}^{-1} \mathrm{~s}^{-1}$, as determined by fitting Eqns 1 and 2 .

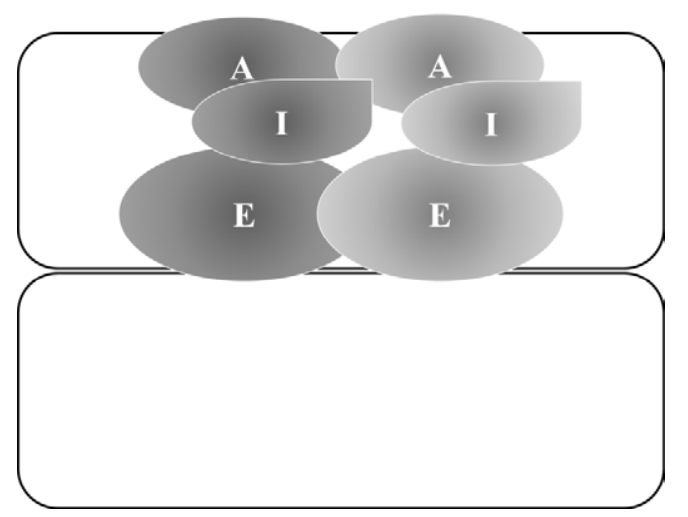

Figure 5: Schematic presentation of GroEL structure showing two rings and 2 subunits consisting of apical $(A)$, intermediate (I), and equatorial $(E)$ domains. The figure is based on the crystallographic data obtained by Braig et al. [15].

inhibits chaperone activity of HSP60 via the covalent binding to Cys442 residue. Remarkably, Cys-442 residue is located in close contact with the ATP-binding pocket (Asp 87) on HSP60 (Nagumo et al., 2005, Supplemental Figure 1), which is a part of the protein's equatorial domain [26]. Our study demonstrates that the interactions between HSP60 and CypD are significantly weakened by the antibody whose immunogen maps to a region between residues 50 and 100 in the equatorial domain. The presence of an invagination in this spot and a number of hydrophobic amino acid side chains potentially makes it suitable for targeting with small molecules.

\section{Acknowledgement}

The author thanks Dr. Milan Stojanovic for providing laboratory equipment and Dr. Steven Taylor for his assistance with the Biacore instrument. The author is grateful to Dr. Francine Katz and Dr. Kung-Ae Yang for scientific discussions and assistance with laboratory work. The author also thanks Dr. Arkadiy Bolshov for valuable discussions. The work was supported by PSC-CUNY research foundation.

\section{References}

1. Gianazza E, Eberini I, Sensi C, Barile M, Vergani L, et al. (2011) Energy matters: mitochondrial proteomics for biomedicine. Proteomics 11: 657-674.

2. Thomas X, Campos L, Mounier C, Cornillon J, Flandrin P, et al. (2005)
Expression of heat-shock proteins is associated with major adverse prognostic factors in acute myeloid leukemia. Leuk Res 29: 1049-1058.

3. 3. Franzén B, Linder S, Alaiya AA, Eriksson E, Fujioka K, et al. (1997) Analysis of polypeptide expression in benign and malignant human breast lesions. Electrophoresis 18: 582-587.

4. Cornford PA, Dodson AR, Parsons KF, Desmond AD, Woolfenden A, et al. (2000) Heat shock protein expression independently predicts clinical outcome in prostate cancer. Cancer Res 60: 7099-7105.

5. Johansson B, Pourian MR, Chuan YC, Byman I, Bergh A, et al. (2006) Proteomic comparison of prostate cancer cell lines LNCaP-FGC and LNCaP-r reveals heatshock protein 60 as a marker for prostate malignancy. Prostate 66: $1235-1244$.

6. Ghosh JC, Dohi T, Kang BH, Altieri DC (2008) Hsp60 regulation of tumor cell apoptosis. J Biol Chem 283: 5188-5194.

7. Cappello F, David S, Rappa F, Bucchieri F, MarasÃ L, et al. (2005) The expression of HSP60 and HSP10 in large bowel carcinomas with lymph node metastase. BMC Cancer 5: 139.

8. Kim HK, Park WS, Kang SH, Warda M, Kim N, et al. (2007) Mitochondria alterations in human gastric carcinoma cell line. Am J Physiol Cell Physiol 293: C761-771.

9. Itoh H, Komatsuda A, Ohtani H, Wakui H, Imai H, et al. (2002) Mammalian HSP60 is quickly sorted into the mitochondria under conditions of dehydration. Eur J Biochem 269: 5931-5938.

10. Agsteribbe E, Huckriede A, Veenhuis M, Ruiters MH, Niezen-Koning KE, et al (1993) A fatal, systemic mitochondrial disease with decreased mitochondrial enzyme activities, abnormal ultrastructure of the mitochondria and deficiency of heat shock protein 60. Biochem Biophys Res Commun 193: 146-154.

11. Magen D, Georgopoulos C, Bross P, Ang D, Segev Y, et al. (2008) Mitochondria hsp60 chaperonopathy causes an autosomal-recessive neurodegenerative disorder linked to brain hypomyelination and leukodystrophy. Am J Hum Genet 83: $30-42$.

12. Lin KM, Lin B, Lian IY, Mestril R, Scheffler IE, et al. (2001) Combined and individual mitochondrial HSP60 and HSP10 expression in cardiac myocytes protects mitochondrial function and prevents apoptotic cell deaths induced by simulated ischemia-reoxygenation. Circulation 103: 1787-1792.

13. McMullin TW, Hallberg RL (1988) A highly evolutionarily conserved mitochondrial protein is structurally related to the protein encoded by the Escherichia coli groEL gene. Mol Cell Biol 8: 371-380.

14. Hohn T, Hohn B, Engel A, Wurtz M, Smith PR (1979) Isolation and characterization of the host protein groE involved in bacteriophage lambda assembly. J Mol Biol 129: 359-373.

15. Braig K, Otwinowski Z, Hegde R, Boisvert DC, Joachimiak A, et al. (1994) The crystal structure of the bacterial chaperonin GroEL at 2.8 A. Nature 371: 578586

16. Jindal S, Dudani AK, Singh B, Harley CB, Gupta RS (1989) Primary structure of a human mitochondrial protein homologous to the bacterial and plant chaperonins and to the 65-kilodalton mycobacterial antigen. Mol Cell Biol 9: 2279-2283.

17. Tanaka Y, Kanai F, Kawakami T, Tateishi K, ljichi H, et al. (2004) Interaction of the hepatitis $B$ virus $X$ protein $(\mathrm{HBx})$ with heat shock protein 60 enhances $\mathrm{HBX}$ mediated apoptosis. Biochem Biophys Res Commun 318: 461-469.

18. Xanthoudakis S, Roy S, Rasper D, Hennessey T, Aubin Y, et al. (1999) Hsp60 accelerates the maturation of pro-caspase-3 by upstream activator proteases during apoptosis. EMBO J 18: 2049-2056.

19. Ban HS, Shimizu K, Minegishi H, Nakamura H (2010) Identification of HSP60 as a primary target of o-carboranylphenoxyacetanilide, an HIF-1alpha inhibitor. J Am Chem Soc 132: 11870-11871.

20. Ghosh JC, Siegelin MD, Dohi T, Altieri DC (2010) Heat shock protein 60 regulation of the mitochondrial permeability transition pore in tumor cells. Cancer Res 70: 8988-8993.

21. Leung AW, Halestrap AP (2008) Recent progress in elucidating the molecular mechanism of the mitochondrial permeability transition pore. Biochim Biophys Acta 1777: 946-952.

22. Fenton WA, Kashi Y, Furtak K, Horwich AL (1994) Residues in chaperonin GroEL required for polypeptide binding and release. Nature 371: 614-619. 
Citation: Korobkova EA (2015) Dynamics of HSP60-Cypd Binding Studied with Surface Plasmon Resonance. Pharm Anal Acta 6: 432. doi:10.4172/21532435.1000432

Page 6 of 6

23. Cheng MY, Hartl FU, Horwich AL (1990) The mitochondrial chaperonin hsp60 is required for its own assembly. Nature 348: 455-458.

24. Jubb H, Higueruelo AP, Winter A, Blundell TL (2012) Structural biology and drug discovery for protein-protein interactions. Trends Pharmacol Sci 33: 241 248.

25. Verdine GL, Walensky LD (2007) The challenge of drugging undruggable targets in cancer: lessons learned from targeting BCL-2 family members. Clin Cancer Res 13: 7264-7270.

26. Nagumo Y, Kakeya H, Shoji M, Hayashi Y, Dohmae N, et al. (2005) Epolactaene binds human Hsp60 Cys442 resulting in the inhibition of chaperone activity. Biochem J 387: 835-840. 